\title{
Del indígena real al indígena imaginario. Crónica de un deseo mestizo
}

\author{
Emmanuelle TREMBLAY \\ Universidad de Quebec en Montreal
}

El indígena es una figura importante de la literatura quebequense, cuyo examen permite interrogar la problemática transcultural. ¿De qué manera sus representaciones actuales nos ilustran sobre la relación de alteridad subyacente en la búsqueda identitaria de la colectividad quebequense? Es lo que se intentará poner de manifiesto, proponiendo un recorrido de lectura que busca desentrañar los componentes simbólicos de lo que aquí llamamos un "deseo mestizo".

Trabajos recientes tienden a revisar la historia social y literaria quebequense, descifrando los signos de una hibridación cultural que se compartiría con la situación continental (véase a Thérien 1992; Van Schendel 1994; Morisset y Waddell 2000). Por ejemplo, el historiador Gérard Bouchard ha propuesto considerar el mestizaje como condición de renovación de un imaginario cristalizado desde el siglo XIX por el mito de la supervivencia francesa en América (2000: 182). Una constante caracteriza al conjunto de estos marcos teóricos. El mestizaje aparece en ellos como un horizonte de cultura posible para pensar una diversidad identitaria que se vio además considerablemente enriquecida por la inmigración en Quebec.

El quebequense es un híbrido cultural ignorado como tal durante mucho tiempo por la historiografía. Heredero de la cultura francesa en América del Norte, se le ha conocido con el nombre de canadiense francés hasta la revolución cultural de los años sesentas. Desde la conquista británica, su identidad se ha consolidado esencialmente respecto al anglosajón, amenaza de asimilación constante. La sociedad canadiense francesa se organizó de este modo de acuerdo con una mitología de la supervivencia del caso francés en el nuevo continente, replegándose en valores de refugio, tales como la raza, la lengua y la religión. Aislado del resto de la realidad canadiense, pero confrontado así en su identidad cultural, el canadiense francés, al igual que el senegalés y el martiniqueño, toma finalmente conciencia de su condición de colonizado, al concebirse como "negro blanco de América" (Vallières 
1974 [1967]). Se afirma entonces como quebequense y declara su aspiración a la independencia nacional.

La amplitud real de la mezcla cultural de la sociedad quebequense con las primeras naciones es difícil de cuantificar, aunque para algunos sea innegable. De Marcel Trudel a Gérard Bouchard, historiadores y sociólogos afirman que, probablemente, hubo un verdadero proceso de mestizaje durante la primera fase de colonización de la Nueva Francia. ${ }^{1}$ De cualquier manera, y contrariamente al caso de América Latina, el mestizaje no ha sido reconocido hasta ahora en Quebec como criterio de definición de identidad. Se trata de un hecho sociológico que se ha convenido en atribuir parcialmente a dos factores: por un lado, al poder tradicional de la Iglesia, y por otro, a la idea de raza. De acuerdo con el primero, el indígena representa la parte del diablo que debe convertirse, así como un freno a la colonización; en cuanto al segundo factor, se apoya en la ideología heredera del conde de Gobineau, que prevaleció a partir de la segunda mitad del siglo XIX para ser transmitida a continuación por el nacionalismo de Lionel Groulx, y que es incompatible con el reconocimiento de un carácter positivo del mestizaje. ${ }^{2}$ Según Fernand Dumont, el indígena "ha ocupado siempre un lugar importante en la simbología de la colectividad de habla francesa": "Los matrimo-

${ }^{1}$ Si bien Marcel Trudel insiste en la aportación amerindia en la composición de la población de la Nueva Francia, en función de la cual "seguramente hubo mestizaje" (1968: 147), Bruce G. Trigger revisó la historia canadiense, a fin de demostrar la parte activa de los amerindios en el comercio de pieles y su impacto en el desarrollo de la colonización del valle del San Lorenzo. En esta última perspectiva, Trigger pone en evidencia los valores culturales que rigieron la interacción entre blancos e indígenas, documentando asimismo sus relaciones sociales, políticas y económicas (1992 [1985]). Para Denys Delâge, dicha interacción se inscribe en el marco de alianzas que son la base de la economía de la Nueva Francia. Es en función de éstas que "los préstamos mutuos, el mestizaje, tienen por efecto difuminar las fronteras [...] sin borrarlas, por supuesto", ya que "el vaivén continuo entre la sociedad colonial francesa y las sociedades indígenas" depende antes que nada del paradigma de la conquista (1991: 23). Sobre las relaciones genealógicas de los canadienses francófonos y anglófonos con lo indígena, ver Smith (1996).

${ }^{2}$ De acuerdo con Bruce G. Trigger, la inferioridad biológica de los indios no entrañaba duda alguna para Groulx. En el contexto de principios del siglo XX, el mestizaje posee por consiguiente un valor negativo. También de acuerdo con Trigger, aun si algunos de los contemporáneos de Groulx criticaron el racismo en la base de su relación con el indígena, "numerosos historiadores prestigiosos, tanto anglófonos como francófonos, siguieron afirmando (y algunos de ellos hasta los años sesentas) que Canadá había tenido la buena fortuna de escapar a las complicaciones que las mezclas raciales entre inmigrantes europeos y pueblos indígenas habían supuestamente ocasionado en América Latina y en otros lugares" $(1992,54)$. 
nios entre indias y colonos, la presencia de los indios al lado de los milicianos en las guerras coloniales, los recuerdos que los viajeros traen de las comarcas lejanas, dejaron legados difícilmente evaluables pero indiscutibles en la colonia" (1993: 68). Es, asimismo, lo que subraya Gérard Bouchard, quien reconoce que la aportación indígena tuvo en un primer momento la función de diferenciación identitaria con la metrópoli francesa, lo cual probablemente permitió a los primeros colonos afirmarse en su experiencia americana. Ahora bien, Bouchard insiste, sin embargo, en la "imagen-ahuyentadora del amerindio torturador y caníbal" que prevaleció más tarde (2000: 87, 8890). Denys Delâge expresó en otros términos la ambivalencia asociada al rol simbólico del indígena: "Creo que tanto el parentesco, aun ficticio, aun exagerado con los amerindios, servía a los canadienses para marcar su identidad frente a los franceses metropolitanos, como el argumento contrario, y sirvió para definir al canadiense frente al británico después de la conquista" (Delâge 1991: 25). Para comprender la problemática identitaria en juego, y más específicamente, para calificar la relación del quebequense con el indígena, es importante, por consiguiente, no prescindir de la complejidad de una situación colonial que, aún en nuestros días, cuenta con ramificaciones en el imaginario colectivo. De hecho, colonizado por su situación económica, política y cultural, el quebequense es, asimismo e históricamente, un colonizador. Se impone así el triángulo de los actores del drama de la alteridad que se plantea desde hace más de dos siglos ya, entre el indígena, el francófono y el anglófono. Estos dos últimos comparten el rol de pueblos fundadores de Canadá, a exclusión del primero, que fue eliminado del reparto histórico. ${ }^{3}$

La relación del quebequense francófono con el indígena implicará, en tales circunstancias, fuertes connotaciones de ambigüedad. ¿Cómo reconocer su papel de colonizador cuando se percibe a sí mismo como colonizado? Lo que quisiéramos subrayar aquí es que, en el plano literario, el indígena

${ }^{3}$ En un artículo en el que se afirma el derecho a la autonomía de las once naciones oficialmente reconocidas por el gobierno de Quebec, Denys Delâge explica la diferencia de situación entre francófonos e indígenas dentro de la confederación canadiense. Los primeros gozan de los derechos cívicos adquiridos como consecuencia de la conquista británica, definidos en función del territorio compartido con los anglófonos. En cuanto a los segundos, sus derechos están determinados según su pertenencia a alguna etnia; su situación es por lo tanto excepcional y encuentra su justificación histórica en el hecho de que ellos eran "aliados" de los británicos, mientras que los canadienses francófonos enseguida se volvieron simples súbditos. Según Delâge, el reconocimiento de tres pueblos fundadores, en lugar de los dos de la historia canadiense, contribuiría a corregir la situación de marginalidad y de tutelaje de las comunidades indígenas (1999). 
remite al héroe quebequense el reflejo de la dualidad que determina su relación con la americanidad y lo confronta con su propia imagen de minoritario. Desde esta perspectiva, el indígena entra en una relación especular que encuentra su manifestación simbólica en la expresión de un deseo mestizo. Lo que también podemos definir, en otros términos, como una manera de interrogar nuevamente a la historia para reconciliarse con el indígena real o con el que, ocultado por el argumento identitario nacional quebequense, vuelve a cobrar importancia en la escena política y social.

Por último, y contrariamente a América Latina, el mestizaje, hasta ahora, no ha sido reconocido culturalmente como un factor de definición identitaria en Quebec. Aunque el indígena no haya sido rescatado por la mitología nacional, no puede menos que considerársele una figura importante del imaginario colectivo. Esto lo han demostrado estudios recientes sobre la concepción narrativa del indígena tanto en las crónicas de la Nueva Francia como en las obras novelísticas y cinematográficas contemporáneas (véase Warwick 1966; Morency 1994; Thérien 1995 y 1986). Desde la perspectiva que aquí adoptamos, la figura del indígena sirve de referencia en el plano cultural. Así, el indígena real, que hoy en día se impone aún en toda su opacidad, sigue siendo considerado como un objeto al que se puede acceder en su diferencia.

\section{Entre dos mundos. Una identidad fronteriza}

En las novelas del primer cuarto del siglo $\mathrm{xx}$, al indígena se le evoca de forma metonímica por la apropiación de sus atributos relacionados con el territorio, en Maria Chapdelaine (1914) de Louis Hémon, y en Le Survenant (1945), de Germaine Guèvremont. Se trata de novelas del terruño que, como todas las del género, escenifican poblaciones blancas sedentarias, cerradas en sí mismas. Sin embargo, en ambos casos encontramos héroes híbridos, pertenecientes a la colectividad canadiense francesa, a la que cuestionan por la atracción que ellos mismos tienen por el gran norte quebequense, o por esta naturaleza "salvaje" que remite a la América autóctona. Trátese de François Paradis en Maria Chapdelaine o del llamado Survenant de Guèvremont, se trata de personajes "indianizados", que encarnan los avatares novelescos del "coureur des bois", ese francés que adoptó el modo de vida autóctono y que fue a la vez, históricamente, un instrumento de la colonización, gracias a su conocimiento del territorio, y el vector para volverse otro.

Es, al menos, de lo que da testimonio el jesuita Charlevoix, quien, desde principios del siglo XVIII, definió al canadiense como un criollo que "vive 
bien con los naturales del país", y que presenta una identidad considerablemente alterada por el Nuevo Mundo. Compartiendo las características físicas y morales del indígena, se distingue en ello del colono inglés, ocupado en el desarrollo del comercio. Si interpretamos a Charlevoix, el canadiense es un hedonista más cerca de la naturaleza que de la cultura. De agradable apariencia personal, se parece al indio por su agilidad (sobre todo para manejar la canoa), lo que le confiere un dominio del territorio, que ha hecho de él un intermediario entre el indio y el comerciante inglés. Intérprete entre el mundo salvaje y el de la civilidad, dividido entre dos identidades, se caracteriza por una libertad individual de la mente y del cuerpo que va contra los imperativos de la supervivencia colectiva y que no fomenta la colonización (Charlevoix 1976 [1744]: 80, 172-173). A semejanza del canadiense de Charlevoix, el "coureur des bois" es algo así como un desertor cultural que prefiere la independencia de la vida errante al trabajo de la tierra. Si bien el héroe de Louis Hémon es leñador en el norte de Quebec a principios del siglo XX, es heredero de esta tradición de la hibridez identitaria y de la marginalidad.

De rostro moreno, vestido en parte a la manera india, François Paradis hace irrupción en el mundo de María Chapdelaine, hija de colonos cuya vida está regida por el ritmo de las estaciones y del trabajo monótono. François vino del norte, llamado comúnmente los "territorios de arriba", zona entonces carente de fronteras y que es también la de la alteridad, fuera de la ley de la pequeña comunidad francófona. Es así como María queda fascinada por François, quien parece "llevar consigo algo de la naturaleza salvaje", y como su deseo toma forma por aquello que, detrás del hombre, indica la presencia autóctona, el narrador nos dice: "A cuatrocientas millas de ahí, en la parte alta de los ríos, los de los 'salvajes' que habían huido de los misioneros y los comerciantes, estaban acuclillados alrededor de una fogata $[. .$.$] y posaban su mirada sobre un mundo aún abundante para ellos$ como en los primeros días de poderes ocultos, misteriosos..." (Hémon 1988 [1914]: 64 y 66)

François Paradis aparece como una figura de mediación entre dos órdenes: el de la cultura, representado por María, y el de la naturaleza. De este modo, la trama del encuentro fundador de las Américas es reactualizada por Louis Hémon. Sin embargo, la modalidad del deseo es introducida en el relato como puente entre la familia católica, representada por María, y el universo pagano cuyo signo es François; María se interpondrá así entre el mito de la supervivencia francesa y la experiencia americana.

El norte quebequense entraña significados simbólicos que se añaden al indígena imaginario tal como éste toma forma en el corpus literario 
quebequense. El indígena remite en realidad a un potencial de libertad que ha sido asociado, históricamente, por Frontenac, gobernador de la Nueva Francia, a una atracción por el "alma del terruño" (citado por Warwick 1966: 272). El amor de María por François no carece de esta connotación espiritual, puesto que está descrito en una terminología relativa a la magia. Víctima de un "sortilegio imperioso" (Hémon 1988 [1914]: 66), venido del norte que la hechiza, María se encuentra encantada por la promesa de volverse otra. Así, el sueño de una "alegría milagrosa" (81) alimentado con el recuerdo de François, está ligado al deseo de unión con ese más allá de la frontera que separa los terrenos de lo conocido y de lo desconocido, de la realidad y de lo posible, del pasado y del futuro, finalmente, podríamos decir, de lo blanco y de lo autóctono.

Bajo estas diversas configuraciones narrativas en la literatura quebequense, el norte sigue siendo ese territorio mítico del Descubrimiento que permite al héroe liberarse de las obligaciones de la civilización y recuperar el paraíso perdido del estado natural. Pues el norte es también el espacio simbólico del viaje y de la libertad sexual, como lo demostró Jack Warwick. De hecho, el nomadismo y la sensualidad definen al "coureur des bois" histórico y son también los rasgos del indígena imaginario (Warwick 1966: 265-293), característicos del héroe de Germaine Guèvremont, en cuya novela se exacerba la relación conflictiva con el territorio de una conciencia deseosa de arraigarse con los suyos, pero que es a la vez incapaz de fijarse en un lugar. De paso en un medio agrícola, el Survenant presenta las cualidades manuales del indígena que va a conquistar el corazón de la sedentaria Angelina. De la misma forma que María por François, Angelina está encantada con el futuro que le ofrece el Survenant, llamado "el salvaje" (Guèvremont 1971 [1945]: 234). Sin embargo, si el héroe de Louis Hémon posee la apariencia física del indígena, el Survenant tiene de éste sobre todo los defectos morales, principalmente la necesidad de embriaguez, ya sea por volverse a marchar o ya por el alcohol, síntoma de una incapacidad para aceptar la obligación y para vivir dentro de las fronteras dispuestas por la moral católica.

De esta manera, estas dos historias de amor (que forman ya parte del folclor quebequense) ofrecen la transposición simbólica de una fascinación por el Otro de la colectividad canadiense francesa, el indígena transportado por la evocación de un "otra parte" inexplorado, aquel que aparece solamente gracias a las figuraciones de la marginalidad. Podríamos desear, por lo demás, que María y Angelina se unieran finalmente a su pretendiente respectivo, para que se produjera, como conclusión, el cruce de los dos mundos representados o una asimilación de uno por el otro. Pero resulta 
imposible considerarlo. Quienes se han vuelto "salvajes" regresan a la naturaleza para desaparecer en ella, cada uno a su manera. Sólo quedan los relatos de un deseo insatisfecho, un deseo mestizo arrancado de su objeto. Es posible ver en esta relación interrumpida una metáfora de las relaciones entre blancos y autóctonos, de un encuentro que no tuvo lugar o de un duelo no asumido del origen mestizo. Debido a sus dos polos identitarios, las múltiples variantes narrativas del "coureur des bois" vienen a paliar esta falta. Por otra parte, su carácter compuesto es el indicio de un posible mestizaje, a pesar de ser portador de la ambigüedad que caracteriza la relación del quebequense francófono con los que Robert Lalonde tan justamente llama nuestros "semejantes diferentes" (1982).

A la vez parecido y diferente, es, efectivamente, bajo esta ambivalencia como el indígena aparece ante el héroe de la ficción contemporánea, sobre todo en Siete lagos más al norte (1993), de Robert Lalonde, y Cowboy (1993), de Louis Hamelin. A la luz de los contenidos simbólicos esclarecidos, es posible ahora examinar brevemente los textos de estos dos autores que comenzaron a publicar en los años ochentas y que han hecho del indígena un personaje central de sus novelas. A pesar de que el indígena tenga en este caso un verdadero papel narrativo, y que la ficción encuentre un anclaje referencial en las reservas autóctonas actuales, sigue perteneciendo al orden de lo imaginario. No obstante, nuevos rasgos vienen a enriquecer esta figura, volviéndola más compleja para informarnos sobre la problemática identitaria del Quebec francófono. Lo que las novelas de Lalonde y Hamelin ponen en escena es un singular punto de vista cultural, una mirada que trata de revelarse a sí misma a través de la alteridad representada.

\section{El buen salvaje y el origen recobrado}

En cuanto al indígena real, sigue estando enmascarado por la imaginería que le da forma. Sin embargo, éste se impuso de manera brutal en 1990, durante un verano que dio lugar a un enfrentamiento armado, en suelo quebequense, entre el ejército canadiense y los iroquenses en Oka. ¿El origen del conflicto? La ampliación de un terreno de golf sobre la reserva de Kanesatake que los mohawk consideran tierras tradicionales. La violencia de tales eventos vino a confirmar, ante los ojos de estupefactos testigos, que América del Norte no había sido conquistada completamente. De hecho, un poder de resistencia que creíamos anestesiado para siempre por eternos procesos jurídicos, dio así testimonio del resurgimiento de la presencia autóctona. No obstante, el argumento de la Conquista fue lo que la crisis de 
Oka reactualizó, en un regreso de la historia sobre sí misma, que obliga a confrontarse con una alteridad radical. Descubrimos así al autóctono frente a la pantalla de nuestra "caja idiota" (para retomar la expresión de Carlos Monsiváis), cerca de barricadas y disimulado tras una máscara en forma de enigma. ¿Qué identidad se encontraba detrás de esta máscara? La pregunta tuvo el efecto de un boomerang: ¿quiénes éramos nosotros para responderla?

Tal es el contexto en el que Robert Lalonde sitúa la acción de su novela publicada tres años después de la crisis que implicaba a los mismos indígenas que se hallaban en Stadaconé cuando llegó Jacques Cartier en 1534, y que debían marcar el imaginario canadiense francés por las torturas infligidas a los jesuitas de la Nueva Francia. Es de hecho con la siguiente imagen como comienza la novela de Lalonde, mientras Michel, el protagonista principal, asiste al drama televisado de la crisis de Oka: "[un] soldado rubio, heroico, limpio, de pie ante su enemigo negro, el mohawk, el guerrero sanguinario [...]" (2000 [1993]: 15). La mitología del farwest es proyectada aquí sobre lo real para hacer de él una ficción basada en la lucha, sin matices, entre cowboys y salvajes. Ahora bien, se perfila a través de este guión de "déjà-vu", el rostro de un indígena en el que Michel reconoce al cómplice de su vida pasada en Oka, el hermano adulado de la infancia, pero también el objeto de un deseo amoroso. El narrador cuenta el regreso de Michel a su pueblo natal, para encontrar el cuerpo del indígena que se esconde detrás del estereotipo guerrero que remite al paraíso perdido del origen autóctono.

La genealogía de Michel incluye una rama iroquense. Mestizo del lado paterno, él es, según una expresión suya, "cuarto de sangre salvaje" (122), un "medio mestizo dividido" (46) entre los dos mundos que coexisten en un mismo territorio en ese país al que su padre llamaba "las tierras robadas", aunque confesaba no saber "quién las había robado a quién" (48). Y ese mismo territorio fue también, durante la crisis de Oka, escenario de un conflicto racial entre las poblaciones blanca y autóctona que lo comparten. Portador él mismo del drama de la conquista, Michel se encuentra, pues, habitado por una violencia fundadora que provoca una escisión en el interior mismo de su ser. El narrador hace además explícita la crisis identitaria que suscitan en él "sus dos naturalezas", "sus dos pieles de nacionalidades", "el rojo y el blanco mezclados": "No quedaba más que este desgarramiento que había originado todo, decidido todo en él desde siempre y para siempre. Doble vida. Polaridades irreconciliables y, sin embargo, unidas, reunidas, abrazadas por fuerza a lo más profundo del cuerpo. Dolor y alegría, unidos desde el comienzo..." (13).

El mestizaje no es pues aquí el principio de una nueva identidad, resultado de un sincretismo unificador. Por el contrario, las diferencias se mantie- 
nen en una relación conflictiva. Así, Michel se nos presenta como el microcosmos de una situación histórica cristalizada por la crisis de Oka, pues la psicología del personaje corresponde a una dualidad irreductible. Resulta importante destacar que la hibridez simbolizada por el personaje del "coureur des bois" y sus avatares en Maria Chapdelaine y Le Survenant se halla interiorizada. Más específicamente, la frontera que separa el mundo blanco de la presencia autóctona toma dos formas. Concebida como un desgarramiento al comienzo del relato, se esfuma a medida que el héroe continúa su búsqueda del indígena en un periplo que termina en la expresión simbólica de una renovación identitaria.

Después de su llegada, Michel emprende un viaje en auto, guiado por un mapa que le ha dejado el indígena, quien se había retirado al bosque. No es casualidad si el trazo de los lugares que recorre Michel corresponde a un ascenso hacia el norte. Como hemos visto antes, éste remite a un espacio de libertad original ligado a la cultura autóctona. La travesía de este territorio mítico permitirá entonces a Michel liberarse de los límites de la civilización. Este recorrido equivale, además, a pasar del otro lado de la frontera que separa su identidad para hallar al indígena en su interior. Este último se manifiesta por medio de descripciones de una naturaleza que despierta la animalidad de Michel, la cual es un rasgo fundamental de la indianidad en la obra de Lalonde, porque lo que hay en el fondo de la búsqueda del héroe es la satisfacción del deseo a través de la apropiación del cuerpo sexuado. Como el narrador lo subraya: "El cuerpo del indígena es todo lo que Michel posee, más grande que él mismo, más fuerte y más vivo que él mismo". "Es el cuerpo del indígena, en su propio cuerpo, él que se apasionó conservando la naturalidad, que amó sin buscar tomarlo todo, sin querer darlo todo" (4243). El cuerpo del indígena, interiorizado por el héroe, es instaurado de este modo como modelo identitario; la componente blanca está, en consecuencia, subordinada y se encuentra alterada en gran medida por este absoluto del deseo.

En su Grand voyage du pays des Hurons (1632), el recoleto Gabriel Sagard dio a conocer sus observaciones sobre las costumbres de los hurones, insistiendo en sus cualidades que juzga moralmente superiores a las de los franceses. La libertad sexual que describe se ha comentado profusamente, hasta volverse un tema asociado al territorio americano, al igual que la fuerza y la belleza natural del cuerpo "primitivo" se convirtieron en los signos de una condición adánica. ${ }^{4}$ En el plano de lo imaginario, el indígena representado

4 “ “...] al anochecer, las jóvenes corren de una cabaña a otra, de la misma manera que, por su parte, lo hacen los jóvenes, que, según sus apetencias, prueban aquí y allá, 
por Lalonde, es heredero de esos lugares comunes sobre el Nuevo Mundo, en la medida en que remite al ideal de un estado natural. En realidad, desde el comienzo del relato se establece una adecuación entre la naturaleza y el amigo de infancia, mientras que la búsqueda de Michel asume igualmente la forma de un ascenso en la memoria en la que el indígena figura como esta otra parte de él mismo que debe ser reconquistada por medio del recuerdo. Así, el deseo del indígena está revestido de un importante valor simbólico, en la medida en que permite la reapropiación identitaria del espacio americano. En este sentido, conserva el mismo papel que tenía en tiempos de la colonización: el de guía. Ahora bien, en este caso, son las fronteras raciales, sexuales y culturales las que este personaje ayuda a franquear para conducir a la conciencia desafortunada del héroe hacia lo que el narrador califica de "promesa increíble" (2000 [1993]: 12). Lo que puede ser asimilado a una voluntad de convertirse en otro al contacto con el cuerpo del indígena o, a lo que podemos llamar, en otros términos, un "deseo mestizo".

Más que simple fantasma de reconciliación, el deseo mestizo invita a interpretar de nuevo la historia del encuentro fundador de la Nueva Francia para que exista una fusión de las identidades presentes. Al final de la novela, el héroe deja los caminos de la civilización y remonta un río para retomar aquí el lugar común de la travesía marítima del Descubrimiento. Como los exploradores antes que él, percibe al indígena en la orilla del río, que es también la de la infancia, ese tiempo mítico de los orígenes donde Michel y el indígena aparecen "lado a lado para olvidar al clan, al pueblo, para sellar el pacto, bajo [un] cielo tranquilo" (26). Finalmente, la situación de conflicto entre blancos y autóctonos da lugar a una posible identidad, sin barricadas, orientada hacia un futuro del mestizaje.

De acuerdo con Gilles Thérien, que dedicó sus investigaciones a demostrar la importancia del indígena imaginario en el plano cultural, sin dejar de distinguirlo del indígena real:

[El personaje del indígena] es a menudo una especie de dios que fue, posiblemente, expulsado de su propio paraíso y que andaba en busca

aunque sin ninguna violencia, dejando las iniciativas a la voluntad de la mujer. El marido hará lo mismo a su vecina, y la mujer al vecino; no interviene ninguna clase de celos entre ellos, y no sienten ninguna vergüenza, infamia o deshonor" (Sagard 1990 [1632]: 200). Portavoz de la naturaleza, sin tuyo ni mío, el indígena ofrece en esta perspectiva, la imagen de la edad de oro de la humanidad que tendrá éxito a partir del siguiente siglo, con el jesuita Joseph-François Lafitau y el barón de Lahontan, incluso con la visión romántica que un Châteaubriand tenía de América. 
de un nuevo comienzo. [...] Representa, en esta sociedad que se pretende moderna, el rechazo de esta modernidad, [ya sea que él] esté consciente de su rechazo o [que] sea su víctima (Thérien 1993: 41).

Éste es otro de los significados del indígena en la novela de Lalonde, porque si el héroe se identifica con este personaje, sólo es para marginarse más respecto a la cultura de consumo. Así, la naturaleza se vuelve, por extensión del cuerpo del indígena, un componente identitario sacrificado al colonialismo económico: "Muerte facturada al progreso, al confort, al irreversible poder del blanco, de su obstinación americana de propietario despilfarrador" (Lalonde 2000 [1993]: 20), para retomar aquí los términos del narrador. $\mathrm{Y}$ es precisamente en este contexto de alteración cultural donde Louis Hamelin sitúa su acción, en una novela en la que el norte quebequense sirve a los intereses del turismo americano. En este caso, es igualmente la trama de la conquista que es reactualizada por el imaginario de la obra. ¿Y qué es lo que hace las veces de Nuevo Mundo?: el paraíso de la caza y de la pesca que administra una compañía para la que trabaja el héroe. En cuanto al conquistador, es el dólar americano que, en este contexto, regula la ley del comercio.

\section{El peligro de volverse otro y la aceptacion de la diferencia}

Gilles Deschênes es un universitario que consigue un empleo de verano como dependiente en un almacén que aprovisiona a la pequeña población ficticia de Grande-Ourse. Él también se encuentra dividido entre dos mundos: el del poder económico que representa el almacén y el de la delincuencia, que caracteriza a la pequeña comunidad india vecina. En realidad, estas dos referencias identitarias están aisladas por un muro de clichés racistas: el blanco y el indígena son, en la obra de Hamelin, vehículos de un odio ancestral que provoca inevitables enfrentamientos, como tantas escenas tributarias de un imaginario a la Sergio Leone. Retirado en la periferia del pueblo, un grupo de desperados bajo la tutela del gobierno hace algunas incursiones a las cercanías del pueblo para tomar cerveza, pelear y saquear el almacén. Aquí, el indígena no tiene nada que ver con el buen salvaje tal como aparece en la obra de Lalonde, aunque comparte ciertos rasgos físicos relacionados con la fuerza y con la destreza por las que es admirado. Lo anima, por el contrario, una venganza que está en el fundamento mismo de la alteridad representada y que permite creer que cada acto de delincuencia no es más que una forma de arreglar cuentas con la historia. 
En semejante contexto maniqueo, el héroe quebequense francófono se convierte en traidor a su comunidad, debido a la fraternidad que lo une a Cowboy, un joven indígena melancólico. Ahora bien, esta identificación con el grupo autóctono es un pretexto para la dramatización de su marginalidad y, sobre todo, de su improductividad en el marco de una sociedad basada en el desarrollo económico. He ahí un rasgo del indígena imaginario en la obra de Hamelin que es, en realidad, un estereotipo de la aprehensión del Otro en las crónicas de la Nueva Francia. Según Marie-Christine GomezGéraud, ser salvaje era, desde un punto de vista capitalista en ascenso, "gastar su energía de manera improductiva". ${ }^{5}$ Al deber elegir entre el espíritu mercantil al servicio del anglosajón y la fiesta de los sentidos que ofrecen las continuas borracheras, el héroe quebequense francófono prefiere volverse salvaje. En este sentido, da al traste con América, al pasar por el campo de los abandonados de la historia, ofreciéndole así una resistencia pasiva, lo que lo llevará por otra parte a experimentar las condiciones de vida de un pueblo que presenta todos los defectos de la civilización.

En la obra de Hamelin, la legendaria libertad del indígena da lugar más bien a la alienación de quien vive de la asistencia social. En tales circunstancias, el héroe se hace guiar, en un mundo en el que la muerte habita los rituales embebidos de alcohol y de nafta, en el que alguien le confiesa no querer llegar a viejo. Pues, explica el narrador, "el problema de la supervivencia se había vuelto cansado para ellos" (1998 [1993]: 312-313). Frente al no futuro del indígena, así como a su desamparo psicológico que sella el suicidio de su amigo Cowboy, Gilles no puede convertirse más que en el testigo de la aculturación de un pueblo en vías de desaparición. También se verá confrontado a una alteridad que cuenta con rasgos distintivos no desprovistos de valor referencial: pobreza, alcoholismo, toxicomanía y violencia bajo todas sus formas, sobre todo las del incesto y de la violación. Finalmente, el desertor se ve rechazado por su comunidad de adopción, durante un episodio que se desarrolla en una reserva autóctona. Ahí, le roban sus papeles, despojándolo así de su identidad. "[P]rivado de [su] definición social", puesto en situación de supervivencia, debe entonces afrontar a los que considera ahora como "una manada inestable" (408) dirigida por un jefe que no reconoce en él más que al enemigo blanco que debe eliminarse.

En un contexto poscolonial que reitera la violencia fundadora de las Américas, la representación de la provincia quebequense permite en esta

${ }^{5}$ La autora se interesó en la representación de la gestualidad en el discurso del colonizador francés. En este contexto, subraya ella, "el hombre del desperdicio, el indio, se ve arrojado a las antípodas de [el homo faber]" (1995: 35). 
novela, de igual modo que en la obra de Lalonde, la concepción de un "deseo mestizo". Aunque este último queda insatisfecho en el caso de Hamelin, ocasiona sin embargo el cruce de las fronteras raciales e ideológicas que permiten al héroe descubrir su propia verdad: la de "la aceptación de la opacidad inalienable del otro", "lo único posible de compartir" (236) entre las identidades presentes. Esta conclusión a la acción novelesca nos permite orientar positivamente el concepto de deseo mestizo en términos de encuentro de culturas y de tensión mantenida por un cruce de miradas que deja la libertad de imaginar al otro en su diferencia.

\section{Conclusión}

Desde hace unos años, las reivindicaciones autóctonas replantean el debate sobre la integridad territorial de la nación quebequense, repentinamente confrontada a los fantasmas de un pasado que creía fosilizado por un folclor de plumas y de tambores (véase Dupuis 1998 [1991]). El indígena real exige actualmente el reconocimiento de sus derechos fundamentales y, sobre todo - como el quebequense-, el de la autodeterminación. Hace, pues, irrupción en una realidad cuyas representaciones son, por lo mismo, cuestionadas. Por otra parte, tales representaciones deben responder a los signos de una nueva coyuntura social que incluya no solamente un reconocimiento territorial y cultural, sino también una nueva problemática identitaria.

El examen de las obras literarias ha permitido extraer contenidos simbólicos para dar forma a lo que podemos concebir como una crónica del deseo mestizo. Ahora bien, la lectura de Lalonde y de Hamelin es igualmente reveladora de la ambigüedad de la relación con el autóctono: orientada hacia la fusión, en el primer caso, exacerba la diferencia en el segundo. Sin embargo, en ambos casos el deseo mestizo funda la representación de la alteridad que pasa por la identificación a lo autóctono. En un contexto de mundialización que algunos han llamado el "siglo americano" (Gruzinski 1999: 9), este deseo se impone como uno de los elementos más significativos. El reconocimiento del deseo mestizo en el plano de lo imaginario y de las figuras de lo mixto, obliga, por supuesto, a plantear de nuevo la cuestión de las mitologías de la identidad, pero principalmente propicia la creación de espacios fronterizos reveladores de las culturas en contacto. Es lo que la creación novelesca permite poner de manifiesto y lo cual sabrán tomar en cuenta, como esperamos, las nuevas narraciones de vida colectiva a las que apelan las formas de la hibridez cultural. 


\section{Obras citadas}

Bouchard, Gérard. 2000. Genèse des nations et cultures du Nouveau Monde. Montreal: Boréal.

Charlevoix, Pierre-François-Xavier de. 1976 [1744]. Journal d'un voyage fait par ordre du roi dans l'Amérique septentrionale. Tercer tomo de su Histoire et description générale de la Nouvelle-France. Ottawa: Éditions Élysée.

Delâge, Denys. 1991. "Les Amérindiens dans l'imaginaire des Québécois". Liberté 33.4-5: 15-28.

1999. "Une souveraineté au pluriel: Les trois peuples fondateurs du Québec". Le Devoir 24 juillet: A9.

Dumont, Fernand. 1993. Genèse de la société québécoise. Montreal: Boréal.

Dupuis, Renée. 1998 [1991]. La Question indienne au Canada. Montreal: Boréal.

Gomez-Géraud, Marie-Christine. 1995. "La perception du geste sauvage et de ses enjeux: regards sur l'Indien de la Nouvelle-France". Figures de l'Indien. Ed. de Gilles Thérien. Montreal: Éditions Typo, 32-45.

Gruzinski, Serge. 1999. La pensée métisse. París: Fayard.

Guèvremont, Germaine. 1971 [1945]. Le Survenant. Montreal: Fides, "Bibliothèque canadienne-française".

Hamelin, Louis. 1998 [1993]. Cowboy. París: Stock.

Hémon, Louis. 1988 [1914]. Maria Chapdelaine. Montreal: Boréal.

Lalonde, Robert. 1982. Le dernier été des Indiens. París: Seuil. 2000 [1993]. Sept lacs plus au nord. Montreal: Boréal.

Morency, Jean. 1994. Le mythe américain dans les fictions d'Amérique. De Washington Irving à Jacques Poulin. Quebec: Nuit blanche éditeur. 
Morisset, Jean y Éric Waddell. 2000. Amériques. Deux parcours au départ de la Grande Rivière de Canada. Montreal: l'Hexagone.

Sagard, Gabriel. 1990 [1632]. Le grand voyage du pays des Hurons. Introd. y notas de Réal Ouellet y Jack Warwick. Montreal: Leméac, "Bibliothèque québécoise".

Smith, Donald B. 1996. "Amerindians in Quebec and Canada, Half-a-Century Ago-and Today". Transferts culturels et métissages Amérique-Europe. $X V I^{e}-X X^{e}$ siècles. Ed. de Laurier Turgeon, Denys Delâge y Réal Ouellet. Sainte-Foy: Presses de l'Université Laval, 117-137.

Thérien, Gilles. 1986. "Les Indiens de celluloïd". Revue d'histoire littéraire du Québec et du Canada français 11: 101-111.

. 1992. "Le métis comme horizon de la disparition". Métissages. Littérature-Histoire. Tome I. Cahiers CRCH-CIRAOI. París: L'Harmattan, 119-130.

. 1993. "Des écrits de la Nouvelle-France à la littérature québécoise". La Licorne 27: 33-46.

,ed. 1995. Figures de l'Indien. Montreal: Éditions Typo.

Trigger, Bruce G. 1992 [1985]. Les Indiens, la fourrure et les Blancs. Français et Amérindiens en Amérique du Nord. Trad. Georges Khal. Montreal: Boréal.

Trudel, Marcel. 1968. Initiation à la Nouvelle-France. Histoire et institutions. Montreal: Holt, Rinehart et Winston.

Vallières, Pierre. 1974 [1967]. Nègres blancs d'Amérique. Montreal: Parti pris.

Van Schendel, Nicolas. 1994. "L'identité métisse ou l'histoire oubliée de la canadianité". La question identitaire au Canada francophone. Récits, parcours, enjeux, hors-lieux. Jocelyn Létourneau y Roger Bernard, ed., Sainte-Foy: Presses de 1'Université Laval, 101-120.

Warwick, Jack. 1966. 'Les 'Pays d'en Haut' dans l'imagination canadiennefrançaise". Études françaises II.3: 265-293. 